\title{
Product of integers in an interval, modulo squares
}

\author{
Andrew Granville \\ Department of Mathematics, University of Georgia \\ Athens, GA 30602-7403 \\ <andrew@math.uga.edu> \\ and \\ J.L. Selfridge \\ Department of Mathematics, Northern Illinois University \\ De Kalb, IL 60115 \\ <selfridge@math.niu.edu>
}

Submitted: September 5, 2000; Accepted: January 15, 2001

\begin{abstract}
We prove a conjecture of Irving Kaplansky which asserts that between any pair of consecutive positive squares there is a set of distinct integers whose product is twice a square. Along similar lines, our main theorem asserts that if prime $p$ divides some integer in $[z, z+3 \sqrt{z / 2}+1$ ) (with $z \geq 11$ ) then there is a set of integers in the interval whose product is $p$ times a square. This is probably best possible, because it seems likely that there are arbitrarily large counterexamples if we shorten the interval to $[z, z+3 \sqrt{z / 2})$.
\end{abstract}

AMS Subject Classification: 05E05 


\section{Introduction}

In optimized versions of several modern algorithms (such as the quadratic sieve), one gradually constructs a set of integers, and tries to efficiently find a (nonempty) subset whose product is a square before the set gets too large. Recently researchers have been analyzing when it is likely that there is a subset of a given set whose product is a square. In [3] Pomerance shows that if we randomly select $\exp (\sqrt{(2+\epsilon) \log x \log \log x})$ integers up to $x$ then, with probability $\rightarrow 1$ as $x \rightarrow \infty$, there is a subset of these integers whose product is a square; whereas if we only have $\exp (\sqrt{(2-\epsilon) \log x \log \log x})$ such integers then the probability $\rightarrow 0$ as $x \rightarrow \infty$. This allows him to give a plausible heuristic to analyze the running time of several important practical algorithms; however, this is only a heuristic since the sets of integers constructed are not really random numbers but rather are determined by some procedure. To unconditionally analyze these algorithms, we need to understand whether there is a subset of certain types of given sets whose product is a square, though this appears to be extremely difficult in the cases of interest.

In this paper we study this type of problem, and variants, where our given set of integers is perhaps as simple as is possible, the integers in a short interval. In a conversation with the second named author in July 1994, Irving Kaplansky conjectured that there is a set of distinct integers, between any pair of consecutive squares, whose product is twice a square. We deduce this as a (trivial) corollary to our

Theorem 1. For every integer $u \geq 2$, there is a set of integers in the closed interval $\left[(u-1)^{2}, u^{2}\right]$ whose product is twice a square.

We conjecture there is a set of at most three integers in $\left[(u-1)^{2}, u^{2}\right]$ whose product is twice a square (see Section 6 for a discussion where we also prove that there is such a set of just two integers for almost all $u$, but not for all $u$ ).

Our proof of Theorem 1 uses the 'Walk method' of [2]. For the interval from 16 to 25, for example, we consider the sequence $5,4,6,3,7,3,8,2,9$. Note that the product of any two consecutive integers in this sequence lies in the closed interval $[16,25]$. Therefore, as we 'walk' along thesequence from 4 to 2 , we get the pairs $4 \times 6,6 \times 3,3 \times 7,7 \times$ $3,3 \times 8,8 \times 2$ giving the integers $24,18,21,21,24,16$ from the interval, whose product is $(4 \times 6)(6 \times 3)(3 \times 7)(7 \times 3)(3 \times 8)(8 \times 2)=4(6 \times 3 \times 7 \times 3 \times 8)^{2} 2=2 \times 6048^{2}$. To deduce Kaplansky's conjecture, we need to cull pairs of the same integer (21 and 24), as well as squares (16), from our sequence $24,18,21,21,24,16$, to obtain the set $\{18\}$. In the proof of Theorem 1 we generalize this method to the interval between any pair of consecutive squares.

Kaplansky's problem is susceptible to various generalizations. For example, when is there a set of integers in $\left[(u-1)^{2}, u^{2}\right]$ whose product is 3 times a square? Or 5 times a square? etc. Alternatively, we might ask for 'large' intervals which do not contain a set of integers whose product is twice a square. We will attack these and related problems in the rest of this article.

Our main theorem is the following:

Theorem 2. Fix the real number $z \geq 10.22$. Suppose that prime $p$ divides some integer in the interval $J=[z, z+3 \sqrt{z / 2}+1)$. Then the product of some subset of the integers 
in $J$, equals $p$ times a square.

If we allow $z$ to run only through integers, then the theorem holds for all integers $z \geq 1$. However, for $z=10.21$ we have $J \subset(10,18)$, and there is no set of integers in this interval whose product is twice a square. An easy consequence of Theorem 2 is that the product of some subset of the integers in $J$ equals $n$ times a square, whenever squarefree $n$ divides the product of the integers in $J$. For, if $a$ and $b$ are coprime, squarefree integers, and $A$ and $B$ are sets of integers such that the product of the elements in $A$ (and in $B$ ) equals $a$ (and $b$, respectively) times the square of an integer, then the product of the elements in $(A \cup B) \backslash(A \cap B)$ equals $a b$ times the square of an integer.

The interval in Theorem 2 cannot be taken to be much shorter as we see from the following examples: If $p$ and $2 p+1$ are both primes, then consider the interval $\left(2 p^{2}-p, 2 p^{2}+2 p\right)$. The only integer in the interval divisible by $2 p+1$ is $(2 p+1) p=2 p^{2}+p$, whereas the integers in the interval divisible by $p$ are $2 p^{2}$ and $(2 p+1) p$. Therefore, no subset of the integers in the interval can possibly have a product equal to $p$ or $2 p+1$ times a square. It is believed that there are infinitely many prime pairs $p, 2 p+1$; and therefore that there are infinitely many primes $p$ which divide an integer in an interval $[z, z+3 \sqrt{z / 2}+3 / 4+O(1 / \sqrt{z})]$, such that no subset of the integers in the interval have product equal to $p$ times a square.

There is an analogous construction with prime pairs $p, 2 p-1$. Theorem 3 below classifies all primes $p$ and intervals $J$ (starting at $z$ with interval length $\geq 5 \sqrt{z / 6}+1$ ), such that $p$ divides some integer in $J$, yet no subset of the integers in the interval have product equal to $p$ times a square. The interval $I_{u}=\left[(u-1)^{2}, u^{2}\right]$, is a subinterval of $[z, z+3 \sqrt{z / 2})$ with $z=(u-1)^{2}$. The primes 911 and 1823 both divide $911 \cdot 1823$, which lies in the interval $\left[1288^{2}, 1289^{2}\right]$, but there is no subset of the integers in this interval which has product equal to 911 times a square. We ask, for which primes $p$ that divide some integer in $I_{u}$, does there exist a set of integers in $I_{u}$ whose product equals $p$ times a square? In Proposition 2 we will see that this is so for any prime $p \leq u^{3 / 4} / 30$, and then show that this is so for any prime $p \leq C u / \log u$, where $C$ is some constant $>0$, assuming:

Conjecture B. There exists some constant $c>0$ such that there is an integer, all of whose prime factors are $\leq \sqrt{x}$, in the interval $[x-c \log x, x]$, for all $x \geq 1$.

As we shall discuss, Theorem 3 below suggests that we should be able to get a good estimate for the number of exceptional $p$ :

Conjecture A. There exists a constant $\kappa>0$ such that there are $\sim \kappa u / \log ^{4} u$ primes $p \leq u$ for which there is no subset of the integers in $I=\left[(u-1)^{2}, u^{2}\right]$ whose product is $p$ times a square.

We can use Theorem 2 to improve our knowledge about a function defined by Erdős in a problem (\#6655) in the American Mathematical Monthly [1]: For each positive integer $n$, define $g(n)$ to be the minimum integer $a_{k} \geq 0$ such that there exists a sequence of integers $n<n+a_{1}<n+a_{2}<\ldots<n+a_{k}$ for which $n\left(n+a_{1}\right) \ldots\left(n+a_{k}\right)$ is a 
square. For example, $g(2)=4, g(3)=5, g(5)=5($ taking $2 \times 3 \times 6,3 \times 6 \times 8,5 \times 8 \times 10$ respectively). Our task is to obtain good estimates for $g(n)$. Define $p(n)$ to be the largest prime which divides $n$ to an odd power. Evidently our sequence of numbers must contain an integer, other than $n$, which is divisible by $p(n)$; since that integer is $\geq n+p(n)$, we must have $g(n) \geq p(n)$. In particular, if $p$ is prime then $g(p) \geq p$. Now if $p>3$ then the interval $(p, 2 p)$ contains an integer that is twice a square, so that $g(p)=p$. For various other integers $n$ we will show that $g(n)=p(n)$. Here we view $g(n)$ as the smallest integer such that there is some set of integers in the interval $(n, n+g(n)]$ whose product equals $n$ times a square.

Corollary 1. For any integer $n$, define $p(n)$ to be the largest prime divisor of $n$. If $p(n)>\sqrt{2 n}+1$ then $g(n)=p(n)$. Otherwise $p(n) \leq g(n) \leq 3 \sqrt{n / 2}+1$.

Proof: If $p=p(n)>\sqrt{2 n}+1$ then write $n=a p$, so that $p>2 a+1$ since $p(p-1)>$ $2 n=2 a p$. Then the product of the integers

$$
n=a p<a(p+1),(2 a+1)(p-1) / 2,(2 a+1)(p+1) / 2,(a+1)(p-1)<(a+1) p
$$

is a square, implying that $g(n) \leq p$. The result follows since we always have $g(n) \geq$ $p(n)$. On the other hand if $p(n) \leq \sqrt{2 n}+1$ then every prime $p$ dividing $n$ satisfies $p \leq p(n) \leq 3 \sqrt{n / 2}+1$ so, by Theorem 2 with $z=n+\epsilon$, there is some set of integers in the interval $(n, n+3 \sqrt{n / 2}+1]$ whose product equals $n$ times a square.

Corollary 1 is close to 'best possible'. For, if $p$ and $2 p+1$ are both prime, with $p>3$, then $g(n) \geq 3 p(n)$ for $n=p(2 p-1$ ) (note that $p(n)=p$ since $2 p-1$ is divisible by 3 ). By

Corollary 1 we have $g(n)<3 p(n)+1$ so $g(n)=3 p(n)(\geq 3 \sqrt{n / 2}+3 / 4)$. One can modify Erdös' problem to ask for $g_{k}(n)$, the minimum integer $a_{k} \geq 0$, such that there exists a sequence of integers $n<n+a_{1}<n+a_{2}<\ldots<n+a_{k}$ for which $n\left(n+a_{1}\right) \ldots\left(n+a_{k}\right)$ is a square. It is easy to determine $g_{1}(n)$ since if $n=r s^{2}$ with $r$ squarefree then evidently $n+g_{1}(n)=r(s+1)^{2}$. Conjecture 3 of [2] states that if $n$ is not a square and $n \neq 8$ or 392 then $g_{2}(n)<g_{1}(n)$. In other words, there exist integers $a, b \in\left(r s^{2}, r(s+1)^{2}\right)$ for which $r a b$ is a square. (Note that if $n=s^{2}$ is a square and $u v^{2}>n$ with $u \geq 1$ then $u(v+1)^{2}>n+2 u v+u>s^{2}+2 s \sqrt{u}+1>(s+1)^{2}$, so $g_{2}\left(s^{2}\right)>g_{1}\left(s^{2}\right)$.) The conjecture is proved in [2, Theorems 4,5,6] except when $r=2$; and in this case except for intervals $\left(2 s^{2}, 2(s+1)^{2}\right)$ where $s=u_{2 m \pm 1} v_{2 m}$ with $u_{m}+\sqrt{2} v_{m}=(1+\sqrt{2})^{m}$. The first two examples here, $u_{1} v_{2}=2$ and $u_{3} v_{2}=14$ yield $n=8$ and $n=392$ respectively.

Acknowledgment: We would like to thank Aaron Meyerowitz and the anonymous referee for their careful reading of this paper.

\section{The Key Proposition}

For integers $a$ and $b$ we write $a \equiv b \quad\left(\bmod \mathbf{Q}^{2}\right)$ if $a / b$ is a rational square; it is easy to show that this is an equivalence relation. Any equivalence class is most naturally represented by the (unique) squarefree integer in that equivalence class. Given an interval $I$, we will denote by $S_{I}$ the set of equivalence classes of products of integers in $I$. Note that $S_{I}$ is closed under multiplication, a fact that we will use repeatedly. 
Proposition 1. Fix the real numbers $1 \leq x \leq y$. Let $I=[x, y+1]$ and $J=[x y, x y+$ $x+y+1)$, unless $x y$ is an integer, in which case we take $J=[x y, x y+x+y)$.

i) For any pair of integers $m<n$ in the interval $I$, there exists some set of integers in the interval $J$ whose product is $m n$ times a square (of an integer).

ii) Suppose that the interval I contains a square. If the product of some subset of the integers in $I$ equals $N$ times a square, then there is some set of integers in the interval $J$ whose product equals $N$ times a square.

Proof: i) Suppose that $a$ is an integer in the range $x \leq a \leq y$, and define $b$ to be the smallest integer $\geq x y / a$ so that $x y / a \leq b<x y / a+1$, and $x y \leq a b<x y+a$. Therefore $(a+1) b=a b+b<(x y+a)+(x y / a+1) \leq x y+x+y+1$ in this range for $a$. If $x y$ is an integer then $a b \leq x y+a-1$, so that $(a+1) b<x y+a+x y / a \leq x y+x+y$. Thus both $a b$ and $(a+1) b$ are in $J$ and therefore in $S_{J}$. But then $a(a+1) \in S_{J}$ since $S_{J}$ is closed under multiplication and $a(a+1) \equiv a b \times(a+1) b \quad\left(\bmod \mathbf{Q}^{2}\right)$. Since $x \leq m \leq n-1 \leq y$ we deduce from the paragraph above that $m(m+1),(m+1)(m+2), \ldots,(n-1) n \in S_{J}$, and so $m n \in S_{J}$ since $m n \equiv m(m+1) \times(m+1)(m+2) \times \ldots \times(n-1) n \quad\left(\bmod \mathbf{Q}^{2}\right)$ and $S_{J}$ is closed under multiplication.

ii) Let $m_{0}$ be a square in $I$, and let $m_{1}, m_{2}, \ldots, m_{k}$ be that subset of the integers in $I$ whose product equals $N$ times the square of a rational number. We may assume that $k=2 \ell$ is even, without loss of generality, for if not, we could remove $m_{i}$ from the list if it equaled $m_{0}$, or add $m_{0}$ to the list if it does not already appear. We may also assume that the $m_{j}$ are distinct (or else we cull any pair of occurences of one number from the list) and so $m_{1}<m_{2}<\ldots<m_{k}$. But then, by i), we have $m_{2 i-1} m_{2 i} \in S_{J}$ for $i=1,2, \ldots, \ell$. Now $N \equiv\left(m_{1} m_{2}\right)\left(m_{3} m_{4}\right) \ldots\left(m_{2 \ell-1} m_{2 \ell}\right) \quad\left(\bmod \mathbf{Q}^{2}\right)$, and thus $N \in S_{J}$, since $S_{J}$ is closed under multiplication.

Proof of Theorem 1: Let $x=u-\sqrt{2 u-1}>1$ and $y=u+\sqrt{2 u-1}$ in Proposition 1, so that $x y=(u-1)^{2}$ is an integer. Let $a$ and $b$ be the smallest positive integers for which $a^{2}, 2 b^{2} \geq x$, so that $(a-1)^{2}, 2(b-1)^{2}<x$, implying that $a-1, \sqrt{2}(b-1)<\sqrt{x}<$ $\sqrt{u}-1 / \sqrt{2}$. Therefore $a^{2}, 2 b^{2} \in I$ since

$$
\begin{aligned}
a^{2} & =(a-1)^{2}+2(a-1)+1<x+2 \sqrt{u} \leq y, \\
2 b^{2} & =2(b-1)^{2}+2 \sqrt{2}(\sqrt{2}(b-1))+2<x+2 \sqrt{2 u}<y+1 .
\end{aligned}
$$

The result follows from Proposition $1(\mathrm{i})$, by taking $\{m, n\}$ to be $\left\{a^{2}, 2 b^{2}\right\}$.

\section{Iterating the key Proposition: The proof of Theorem 2}

Corollary 2. Fix the real number $z \geq(\sqrt{2}-1)^{2}$. Suppose that the product of some subset of the integers in $I=[\sqrt{2 z}-\sqrt{z}, \sqrt{2 z}+\sqrt{z}+1]$, equals $N$ times the square of a rational number. Then there is some set of integers in the interval $J=[z, z+2 \sqrt{2 z}+1$ ) whose product is $N$ times the square of a rational number.

Proof: This follows from Proposition 1(ii) by taking $x=\sqrt{2 z}-\sqrt{z}$ and $y=\sqrt{2 z}+\sqrt{z}$, provided we can show that there is a square in the interval $I$ : If $(\sqrt{2}-1)^{2} \leq z \leq(\sqrt{2}+1)^{2}$ 
then $1^{2} \in I$. If $z>(\sqrt{2}+1)^{2}$ then select $r$ to be the smallest positive integer for which $r^{2} \geq \sqrt{2 z}-\sqrt{z}>1$. Since $r \geq 2$, thus $r \leq 2(r-1)$ and so $r^{2} \in I$ as

$$
r^{2} \leq 4(r-1)^{2}<4(\sqrt{2}-1) \sqrt{z}<(\sqrt{2}+1) \sqrt{z}=\sqrt{2 z}+\sqrt{z}
$$

Lemma 1. Fix the real number $z \geq(\sqrt{2}-1)^{2}$. If $p$ is a prime $\leq \sqrt{2 z}+\sqrt{z}+1$ then there is an integer $k$ such that $p k^{2} \in I=[\sqrt{2 z}-\sqrt{z}, \sqrt{2 z}+\sqrt{z}+1]$.

Proof: If $p \in I$ take $k=1$. Otherwise $p<\sqrt{2 z}-\sqrt{z}$ in which case we select $k$ to be the smallest integer for which $p k^{2} \geq \sqrt{2 z}-\sqrt{z}$; evidently $k \geq 2$. But then $k \leq 2(k-1)$ so that $p k^{2} \leq 4 p(k-1)^{2}<4(\sqrt{2 z}-\sqrt{z})<\sqrt{2 z}+\sqrt{z}$, and the result follows.

Corollary 3. Fix the real number $z \geq 1$. Suppose that $p$ is a prime which divides some integer in the interval $J=[z, z+2 \sqrt{2 z}+1)$. Then there is some set of integers in the interval $J$ whose product equals $p$ times a square.

Proof: If $p \leq \sqrt{2 z}+\sqrt{z}+1$ then, by Lemma 1 , there is an integer $k$ such that $p k^{2} \in$ $I=[\sqrt{2 z}-\sqrt{z}, \sqrt{2 z}+\sqrt{z}+1]$. The result follows from an immediate application of Corollary 2 with $N=p$. If $p>\sqrt{2 z}+\sqrt{z}+1$ then write $m p$ for the smallest integer in $J$ which is divisible by $p$. Evidently

$$
m \leq \frac{z+2 \sqrt{2 z}+1}{p}<\frac{z+2 \sqrt{2 z}+1}{\sqrt{2 z}+\sqrt{z}+1} \leq \sqrt{2 z}+\sqrt{z}+1
$$

so that all of the prime factors of $m$ are certainly $\leq m \leq \sqrt{2 z}+\sqrt{z}+1$. But then, all of the prime factors of $m$ belong to $S_{J}$ (as we saw in the first paragraph of this proof), and so $m$ belongs to $S_{J}$, since $S_{J}$ is closed under multiplication. Moreover $m p \in J$ so that $m p \in S_{J}$, and so $p \in S_{J}$ since $p \equiv m \times m p \quad\left(\bmod \mathbf{Q}^{2}\right)$ and $S_{J}$ is closed under multiplication.

Proof of Theorem 2: For $10.22 \leq z<128$, we proved the result by a computation. For $z \geq 128$, let $I=[x, 2 x+1]$ where $x=\sqrt{z / 2}$. Let $p$ be any prime $\leq 2 x+1$. Note that $p$ divides some integer, call it $m p$, in $I$, for if not then evidently $p<x$, so select integer $a \geq 1$ to be the largest integer for which $a p<x$; then $(a+1) p>2 x$ so that $2 \geq(a+1) / a=(a+1) p / a p>2 x / x=2$ giving a contradiction. Now $x+2 \sqrt{2 x}+1 \leq 2 x+1$ since $x \geq 8$. Therefore the interval $I$ contains an interval of the form $[y, y+2 \sqrt{2 y}+1)$ containing $m p$; and so, by Corollary 3 , there is a set of integers in $[y, y+2 \sqrt{2 y}+1) \subset I$ whose product equals $p$ times a square. We now apply Proposition 1(ii), noting that $I$ contains a square, to deduce that there is some set of integers in the interval $J=$ $\left[2 x^{2}, 2 x^{2}+3 x+1\right)$ whose product is $p$ times a square. Therefore every prime $\leq 2 x+1$ belongs to $S_{J}$. Now suppose $p$ is some prime $>2 x+1$ dividing an integer in $J$. Let's call that integer $m p$, and observe that $m<\left(2 x^{2}+3 x+1\right) /(2 x+1)=x+1 \leq 2 x+1$. Thus every prime factor of $m$ is $\leq 2 x+1$, and so $m \in S_{J}$ (since $S_{J}$ is closed under multiplication). By definition, $m p \in J$ and thus $m p \in S_{J}$; but then $p \in S_{J}$ since $p \equiv m \times m p \quad\left(\bmod \mathbf{Q}^{2}\right)$ and $S_{J}$ is closed under multiplication. 


\section{Classifying the exceptional primes}

Theorem 3. Fix the real number $z \geq 78$, and let $K=[z, z+\Delta]$, where $5 \sqrt{z / 6}+1 \leq$ $\Delta<3 \sqrt{z / 2}+1$, Suppose that prime $\ell$ divides some integer in the interval $K$. There is no set of integers in the interval $K$ whose product equals $\ell$ times a square if and only if one of the following cases holds:

i) There exist primes $p, q, 2 p+1,2 q+1$, one of which is $\ell$, such that $p \geq q$ and

$$
(2 q+1)(p-1)<z \leq 2 p q<p(2 q+1)<z+\Delta \leq q(2 p+2) .
$$

ii) There exist primes $p, q, 2 p-1,2 q-1$, one of which is $\ell$, such that $p \geq q$ and

$$
(2 q-1)(p+1) \geq z+\Delta>2 p q>p(2 q-1) \geq z>q(2 p-2) .
$$

We deduce the following:

Corollary 4. Suppose that for the real number $z \geq 78$, the prime $p$ divides an integer in the interval $J=[z, z+3 \sqrt{z / 2})$, but no subset of the integers in $J$ has product equal to $p$ times a square. Then

Either there exist primes $q, 2 q+1$, one of which is $p$, such that

$$
2 q^{2}-q<z \leq 2 q^{2}<2 q^{2}+q<z+3 \sqrt{z / 2} \leq 2 q^{2}+2 q ;
$$

Or there exist primes $q, 2 q-1$, one of which is $p$, such that

$$
2 q^{2}-2 q<z \leq 2 q^{2}-q<2 q^{2}<z+3 \sqrt{z / 2} \leq 2 q^{2}+q-1 .
$$

Proof of Theorem 3: For $78 \leq z<357$. we proved the result by a computation. So assume $z \geq 357$. Let $x=\sqrt{2 z / 3}$ and $y=\sqrt{3 z / 2}$, so that $x>46 / 3$. Note that $J:=[x y,(x+1)(y+1)) \subseteq K \subset[x y,(x+2)(y+1))$. Any prime $\ell \leq x / 2+1=(y+1)-x$ evidently divides some integer in $I=[x, y+1]$ since the interval is longer than $\ell$. Moreover if $x / 2 \leq \ell \leq(y+1) / 2$ then $2 \ell \in I$, and if $x \leq \ell \leq y+1$ then $\ell \in I$. This accounts for all primes $\ell$ that divide some integer in $I$. Suppose that $\ell$ divides some integer in the interval $I$; since $y \geq x+3 \sqrt{x / 2}>18$ for $x \geq 18$, we see that this integer is contained in some interval $[v, v+3 \sqrt{v / 2}+1) \subset I$ and so $\ell \in S_{I}$ by Theorem 2 . If $18>x \geq 46 / 3$ then $18,24,20 \in I$ so that $2,3,5 \in S_{I}$; moreover if $\ell \geq 7$ and $m \ell \in I$ then $m \ell \leq 28$ so that $m \leq 4$ : thus $m \in S_{I}$ and so $\ell \in S_{I}$. Since $I$ contains a square, we deduce from Proposition 1(ii) that there is some set of integers in the interval $J \subseteq K$ whose product equals $\ell$ times a square. This contradicts the hypothesis, and thus either $\ell \in((y+1) / 2, x)$ or $\ell>y+1$. Suppose that $\ell>y+1$ and it divides $\ell \lambda \in K$. Evidently $\lambda \notin S_{K}$, for if it were then $\ell \in K$ (contradicting the hypothesis) since $S_{K}$ is closed under multiplication. Moreover $\ell \lambda \leq(x+2)(y+1)$ so that $\lambda \leq(x+2)(y+1) / \ell<(x+2)$. Therefore $\lambda$ is prime, otherwise all of its prime factors are $<(x+2) / 2<(y+1) / 2$ and so belong to $S_{K}$, so that $\lambda \in S_{K}$ (since $S_{K}$ is closed under multiplication), giving a contradiction. We also note that $\ell$ then divides only one integer in $K$; otherwise the second such integer would be $\ell(\lambda \pm 1)$ but $\lambda \pm 1$ cannot be a prime since $\lambda$ is, and $2,3 \in S_{K}$.

If $\ell>y+1$ we take $p=\lambda$ (defined as in the paragraph above); otherwise we take $p=\ell$. Therefore $p \in((y+1) / 2, x+2)$ and $p \notin S_{K}$. Note that if $p m \in K$ then 
$m=r$ or $2 r$ for some prime $r$. For, if not then $m=a b$ for some integers $a, b \geq 3$, and $a b p \leq(x+2)(y+1)$, so that $a, b \leq(x+2)(y+1) / 3 p<2(x+2) / 3<(y+1) / 2$. Therefore all of the prime factors of $m=a b$ are $<(y+1) / 2$ and thus in $S_{K}$, so that $m \in S_{K}$ (as $S_{K}$ is closed under multiplication). But then $p \in S_{K}$ since $p m \in S_{K}$, and $p \equiv m \times p m \quad\left(\bmod \mathbf{Q}^{2}\right)$, which contradicts the hypothesis. We also note that $r \notin S_{K}$, for if it were then we would have $m \in S_{K}$, and thus $p \in S_{K}$ (since $S_{K}$ is closed under multiplication). Since $2 p$ is less than $\Delta$, the length of the interval $K$, we see that $p$ divides at least two integers in that interval. In fact $p$ divides exactly two integers in $K$, for if it divided three, call them $p m, p(m+1), p(m+2)$, then one of them must be divisible by 3 , contradicting what we proved in the previous paragraph. Suppose that the two integers in $K$ that $p$ divides are $p m, p(m+1)$. Evidently 2 divides one of $m$ and $m+1$, and we have already seen that these two numbers must each be either prime or twice a prime, so they can be written as $2 q$ and $2 q \pm 1$, where $q$ and $2 q \pm 1$ are both prime but not in $S_{K}$. Since $q \notin S_{K}$ and $q \leq(x+2)(y+1) / 2 p<x+2<y+1$ we can draw the same conclusions for $q$ as we did for $p$ above: that is, $q$ divides exactly two integers in $S_{K}$, namely $2 p q$, and $q(2 p+1)$ or $q(2 p-1)$, where $2 p+1$ or $2 p-1$ (respectively) is prime and not in $S_{K}$ (note that we already knew that $q$ divides $2 p q \in K$ ). We claim that if we have $2 p q, p(2 q+\delta), q(2 p+\epsilon) \in K$ above (where $\delta, \epsilon= \pm 1$ ), then we must have $\delta=\epsilon$ : For, if $q<p$ then $q(2 p+\delta)$ lies between $2 p q$ and $p(2 q+\delta)$ so must be in $K$; similarly if $p<q$ then $p(2 q+\epsilon)$ lies between $2 p q$ and $q(2 p+\epsilon)$ so must be in $K$. Note that either $\ell=p$ or $\ell=2 q+\delta$. We deduce then that $p, q, 2 p+\delta, 2 q+\delta$ must all be prime, and that the only multiples of these primes that belong to $K$ are $2 p q, p(2 q+\delta), q(2 p+\delta)$. To guarantee that these are the only such multiples belonging to $K$ we need to verify that certain inequalities are satisfied. If $\epsilon=1$ these are:

$$
\begin{aligned}
z \leq 2 p q, p(2 q+1), q(2 p+1) & <z+\Delta \\
p(2 q-1), q(2 p-1),(2 p+1)(q-1),(2 q+1)(p-1) & <z \\
p(2 q+2), q(2 p+2),(2 p+1)(q+1),(2 q+1)(p+1) & \geq z+\Delta .
\end{aligned}
$$

Now, by swapping the roles of $p$ and $q$ in the argument above if necessary, we may assume that $p \geq q$. Then we need only check that

$$
(2 q+1)(p-1)<z \leq 2 p q, p(2 q+1)<z+\Delta \leq q(2 p+2) .
$$

A similar argument works when $\epsilon=-1$. It is easy to check that none of the primes $p, q, 2 p+\delta, 2 q+\delta$ belong to $S_{K}$ if $2 p q, p(2 q+\delta), q(2 p+\delta)$ are their only multiples in $K$, since no subset of $p q, p(2 q+\delta), q(2 p+\delta)$ multiplies together to give $p, q, 2 p+\delta$ or $2 q+\delta$ times a square.

Remark: For $z=77.05$ we have $I=[z, z+5 \sqrt{z / 6}+1) \subset(77,96)$. It turns out that all primes that divide some number in $I$, belong to $S_{I}$, except $3,7,13,29,31$.

Proof of Corollary 4: Take $K=J, z \geq 78$ and $\Delta=3 \sqrt{z / 2}$ in Theorem 3, so that either (i) or (ii) there holds. We note that $q=p$, otherwise $q=p-2 a$, for some positive integer $a$. In case (i) this implies that $3 p+1-8 a=4 q+1-p=q(2 p+2)-(2 q+1)(p-1)>\Delta$, 
and in case (ii) this implies that $3 p-1-8 a=4 q-p-1=(2 q-1)(p+1)-q(2 p-2)>\Delta$. Therefore $p>\sqrt{z / 2}+(8 a-1) / 3>\sqrt{z / 2}+2$ and $q>\sqrt{z / 2}+(2 a-1) / 3>\sqrt{z / 2}$. We thus have $z+3 \sqrt{z / 2}=z+\Delta>2 p q>z+4 \sqrt{z / 2}$, giving a contradiction.

\section{The interval $I_{u}=\left[(u-1)^{2}, u^{2}\right]$ revisited}

It is intriguing to determine exactly what primes belong to the set $S_{I}$. When $u$ is small we can easily show that if prime $p$ divides an integer in $I$, then $p \in S_{I}$ : For $u=2$ we have $2=2 \times 1^{2}, 3=3 \times 1^{2}$. For $u=3$ we have $8=2 \times 2^{2}, 6 \times 8=3 \times 4^{2}, 5=$ $5 \times 1^{2}, 7=7 \times 1^{2}$. For $u=4$ we have $10 \times 12 \times 15=2 \times 30^{2}, 12=3 \times 2^{2}, 12 \times 15=$ $5 \times 6^{2}, 10 \times 12 \times 14 \times 15=7 \times 60^{2}, 11=11 \times 1^{2}, 13=13 \times 1^{2}$.

If we assume widely believed conjectures about the distribution of prime pairs, then from Corollary 4, it seems likely that there are infinitely many integers $u$, such that there is some prime $p$ dividing an integer in $I_{u}$, yet $p \notin S_{I}$. Computations in Maple yielded the following prime pairs $p, 2 p+1$ with $p(2 p-1)<(u-1)^{2}<2 p^{2}<p(2 p+1)<u^{2}<2 p(p+1)$ and $u<10^{4}:(u, p)=(1289,911),(3597,2543),(3894,2753),(4191,2963),(4751,3359)$, $(5345,3779),(6779,4793),(7076,5003),(7636,5399),(9961,7043)$. In each case here neither $p$ nor $2 p+1$ belong to $S_{I}$, by Corollary 4 . The construction in Theorem 3(i) can be used here, if there are primes $q<p<2 q+1<2 p+1$ for which

$$
(2 q+1)(p-1)<(u-1)^{2} \leq 2 p q<p(2 q+1)<u^{2} \leq q(2 p+2) .
$$

We consider primes $p$ in the interval [30u/41,5u/7] for which $2 p+1$ is also prime. Then select $q$ to be the largest integer such that $2 q+1<u^{2} / p$. So if $\lambda=u / p$, and $\delta=u^{2} / p-(2 q+1)$ then we need, essentially, $\lambda^{2}-1>\delta>2 \lambda-\lambda^{2}$, which should hold for a positive proportion of such primes $p$. Standard heuristics suggest that the "probability" that $q$ and $2 q+1$ are both prime is $\asymp 1 / \log ^{2} u$. Thus we expect that there should be $\gg u / \log ^{4} u$ such prime quadruplets, and so we propose Conjecture A. On the other hand, we can prove that many primes do belong to $S_{I}$. As an immediate consequence of the following result we see that every prime $p \leq u^{3 / 4} / 30$ belongs to $S_{I}$.

Proposition 2. Let $u \geq 4$ be an integer. If prime $p$ divides some integer in the interval $\left[u-u^{3 / 4} / 30, u\right)$ then there is some set of integers in the interval $I=\left[(u-1)^{2}, u^{2}\right]$ whose product equals $p$ times the square of an integer.

We shall prove this result below after a discussion of what we expect to be true. As we shall see, in the proof of Proposition 2 we show that there exists an integer in any interval $\left[x-3 x^{1 / 4}+1, x\right]$, all of whose prime factors are $\leq 2 \sqrt{x}$. If this could be strengthened as suggested in Conjecture B then we deduce that every prime $p \leq$ $C u / \log u$ belongs to $S_{I}$, for some constant $C>0$ : For if $u^{3 / 4} / 30<p \leq C u / \log u$ then let $x=\left[u^{2} / p\right]$ and select integer $m \in[x-c \log x, x]$, as in Conjecture $\mathrm{B}$, so that all prime factors of $m$ are $\leq \sqrt{x} \leq u / \sqrt{p} \leq u^{3 / 4} / 30$, and so belong to $S_{I}$. Thus $m \in S_{I}$, and $m p \in\left[u^{2}-p\left(1+c \log \left(u^{2} / p\right)\right), u^{2}\right] \subset I$; therefore $p \in S_{I}$. We now proceed to the proof of Proposition 2: 
Corollary 5. Fix an integer $u \geq 4$, and suppose that $p$ is a prime which divides some integer in the interval $J=[u-\sqrt{2 u-1}, u+\sqrt{2 u-1}-1$ ). (In particular any prime $p<2 \sqrt{2 u-1}-1$ divides some integer in the interval.) Then there is some set of integers in the interval $\left[(u-1)^{2}, u^{2}\right]$ whose product equals $p$ times the square of an integer.

Proof: Let $z=u-\sqrt{2 u-1}$, so that $z>1$ and $z+2 \sqrt{2 z}+1=u+\sqrt{2 u-1}-1$. By Corollary 3 we know that there is some set of integers in $J$ whose product equals $p$ times the square of a rational number. The result then follows from Proposition 1(i) by taking $x=z>1$ and $y=z+2 \sqrt{2 z}+2=u+\sqrt{2 u-1}$ (so that $x y=(u-1)^{2}$ is an integer), and noting that in the above proof of Theorem 1 we proved that there is a square in the interval $[x, y+1]$.

Lemma 2. There is always an integer $n$, all of whose prime factors are $\leq 2 \sqrt{x}$, in the interval $\left[x-3 x^{1 / 4}+1, x\right]$ when $x \geq 1$.

Proof: For $x \leq 2000$ we proved the result by direct computation. When $x>2000$ we select $a$ to be the smallest integer $\geq \sqrt{x}$, and then $b$ to be the smallest positive integer $\geq \sqrt{a^{2}-x}$. We find that $a<1+\sqrt{x}$, so that $a^{2}-x<1+2 \sqrt{x} \leq \frac{1}{4}\left(3 x^{1 / 4}-1\right)^{2}$, and thus $b-1<\sqrt{a^{2}-x}<\left(3 x^{1 / 4}-1\right) / 2$. Let $n=a^{2}-b^{2}=(a-b)(a+b)$, so that the prime factors of $n$ are $\leq a+b<1+\sqrt{x}+1+\left(3 x^{1 / 4}-1\right) / 2<2 \sqrt{x}$. Moreover $x-n=b^{2}-\left(a^{2}-x\right)$ so that, by definition of $b, 0 \leq x-n \leq 2(b-1) \leq 3 x^{1 / 4}-1$.

Proof of Proposition 2: The result follows directly from Corollary 5 in the range $4 \leq$ $u \leq 3 \times 10^{6}$ since then $u^{3 / 4} / 30<\sqrt{2 u-1}$. We may thus assume that $u>3 \times 10^{6}$.

Suppose that $p$ divides $u-a$ where $a$ is a positive integer $\leq u^{3 / 4} / 30$. If $p \leq \sqrt{u}$ then we know that $p \in S_{I}$ by Corollary 5 . If $p>\sqrt{u}$ then $(u-a) / p \leq \sqrt{u}$ and so belongs to $S_{I}$. Thus, since $S_{I}$ is closed under multiplication, we see that $p \in S_{I}$ if and only if $u-a \in S_{I}$. Note that the result follows from Corollary 5 if $a \leq \sqrt{2 u-1}$; so we assume henceforth that $a>\sqrt{2 u-1}$. Let $n$ be the largest integer $\leq 3 u^{1 / 4}-1$. By Lemma 2 there are integers, in both of the intervals $[u-a-n, u-a]$ or $[u-a, u-a+n]$, which have all of their prime factors $\leq 2 \sqrt{u}$ (we will call such an integer $u-b$ below). By Corollary $5 u-b \in S_{I}$. We shall show that for one of these choices of $u-b$, we have $(u-b)(u-a) \in S_{I}$. Thus $(u-a) \in S_{I}$ (and so $p \in S_{I}$ ), since $S_{I}$ is closed under multiplication. Select $k$ to be the greatest integer $\leq a^{2} /(u-a)$ so that $u^{2}-(u-a)<(u-a)(u+a+k) \leq u^{2}$. We note that $n+1 \leq 3 u^{1 / 4}$ and $k \leq u^{1 / 2} /\left(900-30 u^{-1 / 4}\right) \leq u^{1 / 2} / 870$. If $(u-a)(u+a+k) \geq u^{2}-\frac{u-a}{2}$ then for $A=a, a+1, \ldots, a+n$ we have

$$
\begin{aligned}
u^{2} & \geq(u-a)(u+a+k) \geq(u-A)(u+A+k)>(u-A-1)(u+A+k) \\
& \geq(u-a-(n+1))(u+a+k+n) \geq u^{2}-\frac{3(u-a)}{2}-(n+1)(2 a+k+n) .
\end{aligned}
$$

Now

$$
(n+1)(2 a+k+n)<3 u^{1 / 4}\left(\frac{u^{3 / 4}}{15}+\frac{u^{1 / 2}}{870}+3 u^{1 / 4}\right) \leq \frac{u}{5}+\frac{u^{3 / 4}}{290}+9 u^{1 / 2}<\frac{u}{2}
$$


for $u \geq 904$ and so the lower bound above is $\geq(u-1)^{2}$. Therefore $(u-A)(u+A+k)$ and $(u-A-1)(u+A+k)$ both belong to $I$ and so to $S_{I}$. Multiplying these together gives $(u-A-1)(u-A) \in S_{I}$; and then multiplying together this result for $A=a, a+1, \ldots, b-1$ to get that $(u-b)(u-a) \in S_{I}$ and the result follows. If $(u-a)(u+a+k) \leq u^{2}-\frac{u-a}{2}$ then note that $(u-a)(u+a+k-1)=(u-a)(u+a+k)-(u-a)>u^{2}-2(u-a)>(u-1)^{2}$. Thus for $A=a, a-1, \ldots, a-n$ we have

$$
\begin{aligned}
(u-1)^{2} & \leq(u-a)(u+a+k-1) \leq(u-A)(u+A+k-1) \\
& <(u-A+1)(u+A+k-1) \leq(u-a+(n+1))(u+a+k-(n+1)) \\
& \leq u^{2}-\frac{u-a}{2}+(n+1)(2 a+k-(n+1)) .
\end{aligned}
$$

Now, proceeding as above, we have

$$
(n+1)(2 a+k)+a / 2<\frac{u}{5}+\frac{7 u^{3 / 4}}{348}<\frac{u}{2}
$$

for $u \geq 923$ and so the upper bound here is $\leq u^{2}$. Therefore $(u-A)(u+A+k-1)$ and $(u-A+1)(u+A+k-1)$ both belong to $I$ and so to $S_{I}$. Multiplying these together gives $(u-A)(u-A+1) \in S_{I}$; and then multiplying together this result for $A=b+1, \ldots, a$ to get that $(u-a)(u-b) \in S_{I}$ and the result follows.

\section{Minimal sets whose product is twice a square}

We consider the smallest set of integers $S \subset I_{u}$ whose product is twice a square:

Suppose that $|S|=1$ : That is, there exists an integer $m$ such that $(u-1)^{2}<$ $2 m^{2}<u^{2}$. This is equivalent to the fractional part of $u / \sqrt{2}$ being $<1 / \sqrt{2}$, which occurs for $\sim U / \sqrt{2}$ of the integers $u \leq U$.

Suppose that $|S|=2$ : That is, there exist integers $g, m, n$, with $g$ odd and squarefree, such that $(u-1)^{2} \leq 2 g m^{2}, g n^{2} \leq u^{2}$. (The $|S|=1$ case is just the case $g=1$ here.) We checked this, for given $u$, by taking each odd and squarefree $g \leq 2 u$ and then determining whether there are integers $m$ and $n$ with $(u-$ $1)^{2} \leq 2 \mathrm{gm}^{2}, g n^{2} \leq u^{2}$. There are 123 exceptional values of $u$ up to $10^{4}$, namely $4,14,21,79,86,93,100, \ldots, 7368,7423,7846,8044,8758$. Now, for a fixed $g$, there exists an integer $n$ for which $(u-1)^{2}<g n^{2}<u^{2}$, if and only if $\{u / \sqrt{g}\}<1 / \sqrt{g}$, where $\{t\}$ denotes the fractional part of $t$. If we randomly choose a value of $u \leq U$, then the probability that this happens for one given odd, squarefree value of $g$ is $\sim 1 / \sqrt{g}$. By ergodic theory we know that such probabilities are independent so that the 'probability' that a randomly chosen value of $u$ satisfies $\{u / \sqrt{g}\}<1 / \sqrt{g}$ and $\{u / \sqrt{2 g}\}<1 / \sqrt{2 g}$ simultaneously is $1 / g \sqrt{2}$. Indeed, for any fixed $G$, we can prove that the number of integers $u \leq U$ for which there is no triple $g, m, n$ satisfying $(u-1)^{2} \leq 2 g m^{2}, g n^{2} \leq u^{2}$, where $g \leq G$ is odd and squarefree, is $\sim U \prod_{g}(1-1 / g \sqrt{2})$ where the product is over odd, squarefree integers $g \leq G$. Now, it is easily shown that $\prod_{g}(1-1 / g \sqrt{2})=G^{-2 \sqrt{2} / \pi^{2}+o(1)}$ 
as $G \rightarrow \infty$. Thus there are $o(U)$ exceptional $u \leq U$. If we were to suppose that our formula held with appropriate uniformity (i.e. taking $G=2 U$ above) then we'd expect that the number of integers $u \leq U$, such that there are no two integers in $\left[(u-1)^{2}, u^{2}\right]$ whose product is twice a square, is $U^{1-2 \sqrt{2} / \pi^{2}+o(1)}$, and we note that this exponent is $.71342041 \ldots$ We'd thus expect about 138 such integers $u \leq 10^{4}$, whereas we found above that the correct number is 123 , so our heuristic is more-or-less borne out in practice.

Scott Contini then wrote a program checking that for each $u$ in the above list, there do exist three numbers in $\left((u-1)^{2}, u^{2}\right)$ whose product is twice a square; for examples, $3^{2}<2 \times 5<3 \times 2^{2}<3 \times 5<4^{2}$, then $13^{2}<19 \times 3^{2}<5 \times 6^{2}<2 \times 5 \times 19<14^{2}$, and $8757^{2}<2 \times 11 \times 1867^{2}<7 \times 11 \times 998^{2}<7 \times 3310^{2}<8758^{2}$. Thus we can conclude that there is a nonempty set of integers, with no more than three elements, in any $I_{u}$ for $u<10^{4}$, whose product is twice a square. Presumably this is true for all $u \geq 2$.

\section{References}

[1] P.T. Bateman, P. Erdős, J.L. Selfridge, Getting a square deal, Amer. Math Monthly 99 (1992) 791-794.

[2] P. Erdős, J.L. Malouf, J.L. Selfridge and E. Szekeres, Subsets of an interval whose product is a power, Disc. Math. 200 (1999), 137-147.

[3] C. Pomerance, The role of smooth numbers in number theoretic algorithms, Proc. Int. Cong. Math., Birkhäuser Verlag, Basel (1994), 411-422. 